\title{
Suppressing prompt splash with polymer additives
}

\author{
E. J. Vega • A. A. Castrejón-Pita
}

Received: date / Accepted: date

\begin{abstract}
Splash suppression during drop impact continues to be a grand challenge. To date, only a few techniques for the complete suppression of splash exist. Reducing the ambient pressure and using complex surfaces (microstructured and/or soft) are two of the recently discovered ones which may not be very practical in many technological processes. The idea of using additives directly into the liquid used to produce the drops, in order to inhibit this undesirable phenomenon, is therefore desired. Prompt splash is a type of splashing that releases diminutive droplets at high speeds from the tip of the lamella at the spreading liquid-substrate contact line immediately after the impact (within the first $10 \mu \mathrm{s}$ ), without generating the typical thin-sheet or corona. Prompt splash remained hidden for many years until high-speed imaging allowed for its visualisation. Here we demonstrate that by adding very low amounts of polymer (around 0.01 wt \%) into normally splashing water droplets a reduction and even a complete suppression of the prompt splash is observed. In this work, a systematic experimental study of the impact of viscoelastic drops, by varying size, impact velocity,
\end{abstract}

E. J. Vega

Depto. de Ingeniería Mecánica, Energética y de los Materiales and Instituto de Computación Científica Avanzada (ICCAEx),

Universidad de Extremadura,

Avda. de Elvas s/n, E-06006 Badajoz, Spain

Tel.: +34-924-289600

Fax: +34-924-289601

E-mail: ejvega@unex.es

A. A. Castrejón-Pita

Department of Engineering Science, University of Oxford, Parks Road, Oxford OX1 3PJ, United Kingdom

Tel.: +44 (0)1865 273019

Fax: +44 (0)1865 273010

E-mail: alfonso.castrejon-pita@eng.ox.ac.uk and the "degree" of viscoelasticity, is conducted. When capillary forces are insufficient to maintain the integrity of the drop, elastic forces seem to pull the attached small droplets/fingers back to the lamella preventing their ejection, and therefore, inhibiting prompt splash. However, surprisingly larger quantities of the polymer additive lead to a secondary transition, in which another, more common, type of splash is induced: corona splash.

Keywords splash $\cdot$ polymer additives $\cdot$ prompt splash suppression

\section{Introduction}

The phenomenon of a liquid droplet impacting on a solid surface is of fundamental importance due to its appearance in innumerable applications: inkjet printing [1], delivery of agrochemicals and pharmaceuticals [2], spray coating for food [3] or pharmaceutical [4] industry, fabrication of microlenses [2], as well as spray painting and cleaning, spray cooling and firefighting devices, soil erosion due to rain drop impact, fuel atomization in internal combustion engines, turbine wear, and many other. Extensive literature on the dynamics of impacting and spreading/receding Newtonian liquid drops can be found, as shown in the complete reviews of Yarin [5] and Josserand et al. [6], but the studies about the impact of non-Newtonian liquid droplets on a solid surface are quite more limited [7].

The spreading and receding behaviours have been studied for drop impact of shear-thinning [8,9], shearthickening [10], and yield-stress [9] liquids. The maximum diameter of the spreading is a parameter of great interest $[11,12]$ as it allows the optimisation of liquid 

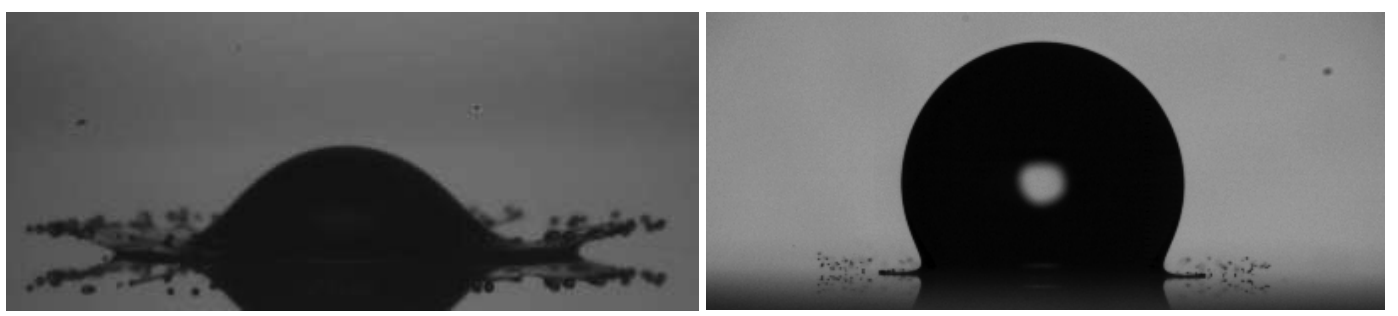

Fig. 1 Corona splash (left) and prompt splash (right)

coverage (e.g. for thin film manufacture or inkjet printing). The hydrophilicity, hydrophobicity and roughness/ micro-structure of the surface can greatly influence the outcome [13]. Moreover, during the receding stage, rebound phenomena can appear under certain impact conditions. The improvement of adhesion onto hydrophobic surfaces after droplet impact (anti-rebound effect) is a key characteristic in many technologies e.g., for the effective delivery of agrochemicals [14,15]. The addition of a very small (dilute) amounts of polymeric additives has been shown as a solution to inhibit the rebound effect [16-24]. Energy dissipation caused by the addition of polymer additives in the spreading phase seems to be insignificant. However, the effects during the capillary retraction of the drop are considerable. This dissipation has traditionally been attributed to an increased extensional viscosity as the polymers stretch in the extensional flow of the droplet [16,17]. Smith et al. [18-20] demonstrated, however, that the observed anti-rebound effect is due to the stretching of polymer molecules as the droplet edge sweeps the substrate, slowing down the movement of the receding contact line. This modification in the receding contact angle dynamics has been supported elsewhere $[22,23]$. In a recent work, Hub et al. [24] added more reasons of this extra-dissipation, asserting that the energy dissipation caused by the presence of polymer additives is a complex function of the concentration and molecular weight of the polymer solution, the contact-line retraction velocity, and the area of polymer residue (left on the surface during the previous spreading phase). Surprisingly, in contrast with the mentioned anti-rebound effects of polymeric additives, a recent computational study of Izbassarov et al. [25] shows that the viscoelasticity enhances the tendency of the drop to rebound in the receding phase. These results suggest that the anti-rebound effects are mainly due to the polymer-induced modification of wetting properties of the substrate rather than the change in the material properties of the liquid of the drop. One can conclude that this anti-rebound effect still needs further study.

With regards to the influence of a polymer additive on the splashing, i.e., when the drop hitting a solid surface disintegrates into smaller satellite droplets because the capillary forces are insufficient to maintain its integrity, just a few works can be found in the literature. Crooks et al. [26] observed that the splash threshold of the elastic drops was significantly higher than that of Newtonian drops and that threshold increased for greater molecular weights of polymer. In experiments of drops of polymer solutions impacting on small solid targets, it was mentioned that the polymeric additives increase the "strength" of the liquid preventing the rupture of the liquid lamella [27]. Finally, on a hot surface, the additive was shown to inhibit drop splashing too [28-30], attributing this behaviour to the large energy dissipation caused by stretching of polymer chains in elongational flows during the radial expansion of the lamella. It is important to note that the cited experimental studies were conducted by capturing images at around 1000 frames per second (the so-called highspeed imaging at the time), so due to the early imaging limitations, i.e., the slow frame rates used, a quite faster type of splash, such as "prompt" splash, could not be observed.

While the exact physical mechanisms underlying the dynamics of splash is still unclear [6], two types of splash on solid surfaces are observed: corona (thin-sheet) and prompt [31-33], illustrated in Fig. 1. In the corona splash the intact lamella is separated from the substrate forming a thin sheet that eventually reaches a corona shape (bowl-like structure) ejecting secondary small droplets from the tip of finger-like structures, whereas a prompt splash releases very fast small droplets from the tip of lamella at the spreading liquid-substrate contact line immediately after the impact, without the presence of the typical thin-sheet/corona structure. It is theorized that corona splashing is generated by the interplay between the fast liquid ejecta, the surrounding fluid, and the properties of the fluid (competing roles between viscosity and surface tension). Prompt splash on the other hand arguably appears due to to the roughness of the substrate. However, this assumption seems to offer an incomplete picture as prompt microsplashing is observed for impacts on to smooth surfaces [34] and therefore the mechanisms underlying prompt splash are not yet clear. 
In the last decade, splashing has been inhibited by using different techniques. By decreasing the air pressure, droplet ejection is inhibited or even suppressed not only during thin-sheet formation but also for prompt splashing [32]. Another useful technique for splash suppression is by carefully modifying the roughness/microstructure of the solid substrate. Liu et al. [35] showed that a strategically placed ring of $75 \mu \mathrm{m}$ through-holes in the substrate was enough to suppress the splash due to the drainage of the air layer under the outer edge of the lamella. More recently, Howland et al. [36] demonstrated that the substrate stiffness can also affect the splashing threshold, by reducing the local pressure in and around the contact region during drop impact, hence reducing the speed the of the lamella, resulting in a reduction of the splash. All these techniques involve the change of either the properties of the surrounding fluid or the target surface, but can prompt splash be inhibited by directly using additives in the drop impacting?. To the best of the authors' knowledge, this question has not been answered yet.

In this work, a systematic experimental study of the impact of viscoelastic drops, with different sizes, speeds, and "degrees" of viscoelasticity, is conducted in order to answer the question set out above. We will show that a very low concentrations of polymer in water (around $0.01 \mathrm{wt} \%$ ) are enough to practically suppress prompt splash. Elastic forces pull the would-be small droplets back into the ejecta, keeping the integrity of the liquid configuration.

The main dimensionless groups characterizing our system are: Weber number $W e$, Deborah number $D e$, Reynolds number $R e$, and the ratios of the properties of the two fluids involved (liquid-gas) $\rho / \rho_{\text {air }}$ and $\mu / \mu_{\text {air }}$, densities and viscosities respectively [37]. When a drop impacts on a solid surface, Re and $W e$ balance the inertia with the viscous and the capillary forces, respectively, whereas $D e$ takes the fluidity (elasticity and viscosity) of the drop into account during the impact dynamics, i.e., the polymeric contribution. $W e=$ $\rho D u_{0}^{2} / \sigma$, where $\rho$ is the density, $D$ the diameter of the impacting drop, $u_{0}$ the normal velocity of impact of the drop, and $\sigma$ the surface tension. We ranged from 200 to 1100 in our experiments. De is defined as the ratio between the relaxation time of the working liquid $\lambda$ and the characteristic impact time $t_{0}=D / u_{0}$, i.e., $D e=\lambda / t_{0}$, varying within the range of 0-200 in our experiments. The Reynolds number is defined in terms of the infinite-shear viscosity $\mu_{\infty}, R e=\rho D u_{0} / \mu_{\infty}$, because the shear rate $\dot{\gamma}$ is expected to be very high in our experimental runs, so Re ranges approximately from 1200 to 24000 . The ratio $\rho / \rho_{\text {air }}$ is kept constant, and the ratio of viscosities, taking the infinite-shear viscos- ity of the liquid, $\mu_{\infty} / \mu_{\text {air }}$, varies from 55 to 295 . Therefore, in this study, we will systematically vary $W e, D e$, and $R e$ in order to understand their role on the impact dynamics and its outcome.

The manuscript is organized as follows. In Sec. 2 the experimental apparatus and working fluids are described together with the experimental procedure. The experimental data are presented and analyzed in Sec. 3. The paper closes with the main discussion and conclusions in Sec. 4.

\section{Materials and methods}

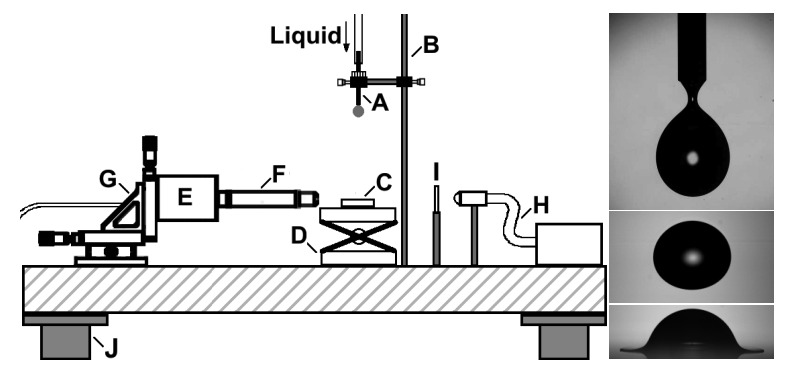

Fig. 2 (Left) Experimental setup: capillary/needle (A) fixed on vertical support (B), target substrate (C), platform (D), digital camera $(\mathrm{E})$, optical lenses $(\mathrm{F})$, triaxial translation stage $(\mathrm{G})$, optical fiber $(\mathrm{H})$, optical diffuser (I), and optical table $(\mathrm{J})$. (Right) Illustrative evolution of the drop: formation, flight, and impact.

An sketch of the experimental setup is shown in Fig. 2 (left). In order to form the drops, the working liquid was injected slowly through a steel capillary/needle (A) forming a pendant drop that eventually detached under its own weight. The capillary/needle could be displaced along a vertical support (B) in order to control the height over the target substrate $(\mathrm{C})$ placed on a platform (D), ranging from 20 to $120 \mathrm{~cm}$ in our experiments. This height set the normal impact velocity $u_{0}$ which was varied between 2.2 and $4.8 \mathrm{~m} / \mathrm{s}$. The target substrate consists of a clean glass slide (SELECT Micro SLIDES), with a typical root-mean-square roughness of a few nanometres. After each impact event, the smooth solid surface was either carefully cleaned and dried or exchanged in order to prevent contamination from previous deposited drops. Experiments were conducted at atmospheric pressure and at $24 \pm 2{ }^{\circ} \mathrm{C}$.

Digital images of the impact of each single drop were acquired by using an high-speed CMOS camera (Phantom V.12.1) (E) equipped with a set of optical lenses/microscope (F). Two different optical acquisition parameters and magnifications were used: most 
images were taken at 44000 frames per second with an exposure time of $1 \mu \mathrm{s}$ and a magnification of 20.83 $\mu \mathrm{m} /$ pixel, and zoomed-in images were taken at up to 360000 frames per second with an exposure time of 285 ns and a magnification of $6.31 \mu \mathrm{m} /$ pixel. The camera could be displaced both horizontally and vertically by using a triaxial translation stage $(G)$ in order to focus directly into the region of the drop impact and contact line. The impact events were back-illuminated with a cool white light, provided by an optical liquid fiber light source (Photofluor II) (H). A micro-machined optical diffuser (I) was positioned between the optical fiber and the object to provide a uniformly lit background. All these elements were mounted on an optical table (J). By the way of illustration, Fig. 2 (right) shows three images acquired in the course of the experiments, showing the three main stages of the drop's lifetime: formation, flight, and impact.

The working fluids utilized during the experiments consisted of pure distilled water with small quantities of polymeric solutions of polyacrylamide (PAA) (Polysciences, $\left.M_{w}=18 \times 10^{6} \mathrm{~g} / \mathrm{mol}\right)$. Stock solutions with concentration $c$ were prepared by dissolving the polymer in the solvent by agitation with a magnetic stirrer at very low angular speeds in order to avoid both the formation of undissolved polymer clusters, which could result in inhomogeneous solution, and the breakage of the polymer chains. The PAA concentrations ranged between 0 (pure water) and 1000 ppm.

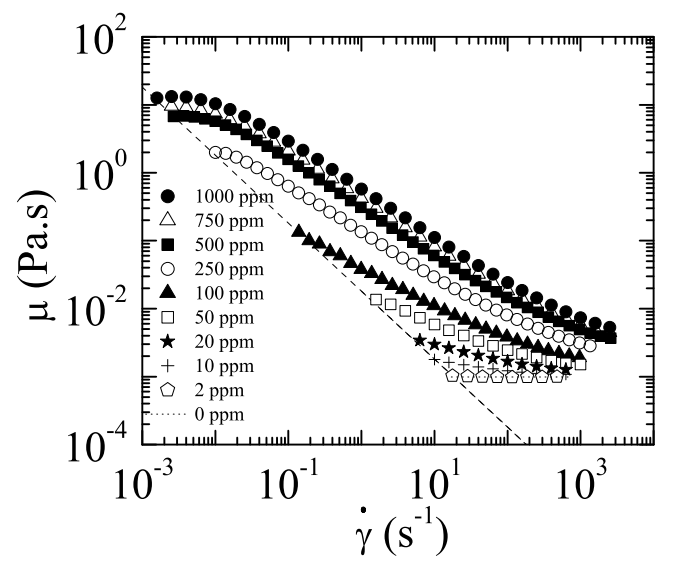

Fig. 3 Dependence of the solution shear viscosity $\mu$ upon the shear rate $\dot{\gamma}$ for the PAA solutions with concentrations $c$ of PAA in water between 2 and 1000 ppm. The starting points of the curves are determined by the rheometer sensitivity. The dotted horizontal line represents the shear viscosity of the solvent [38].

The dependence of the shear viscosity $\mu$ upon the shear rate $\dot{\gamma}$ was measured with a rheometer PHYSICA
MCR 301. The results are displayed in Fig. 3. Shearthinning in the PAA solutions becomes more pronounced when the polymer concentration increases. This effect is more noticeable for $c>50 \mathrm{ppm}$, close to the overlap concentration $c^{*}=45 \mathrm{ppm}$ [39]. Therefore, the polymer solutions used in the present work cover both the dilute and the semi-dilute regime. The polymer relaxation time $\lambda$ was measured with a new setup for filament breakup technique, based on the slow retraction method using high-speed imaging visualisation and an immiscible oil bath to reduce solvent evaporation [38]. In Table 1 the relevant values for all the polymeric solutions are listed.

Finally, the surface tension $\sigma$ was measured with the TIFA method [40]. We verified that, $\sigma \simeq 72 \mathrm{mN} / \mathrm{m}$ and $\rho \simeq 997$ in all the cases. These fluid properties were, within experimental error and within the studied range of concentrations, identical to those of pure water.

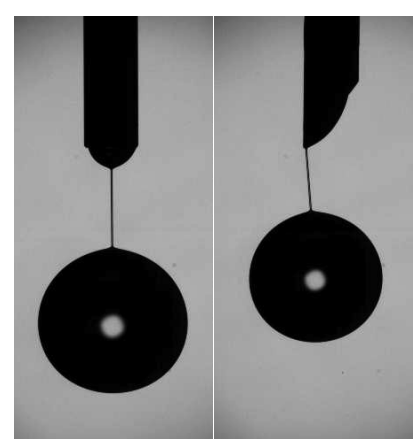

Fig. 4 Drop produced with $c=750 \mathrm{ppm}$ detaching from a standard capillary (left) and beveled needle (right), both feeding elements with a outer diameter of $1.27 \mathrm{~mm}$.

In our experiments, five different feeding elements were used in order to form the drop, taking into account the influence of the drop size, i.e., the role of Reynolds number $R e$, as it will be shown in Sec. 3. Table 2 shows the parameters of each element and the drop resulting. It was checked that pure water drops below $3 \mathrm{~mm}$ in diameter $D$ showed hardly any prompt splash for heights around the maximum realistically allowed by our experimental setup. On the other hand, for drop diameters $D$ above $5 \mathrm{~mm}$ the oscillations of the drops during the flight were large enough to see its influence on the shape of the drop just before the impact and on the outcome of the impact itself [41-43].

A beveled needle was used in the experimental run including the highest concentration of our semi-dilute polymer solutions in order to minimise the breakup time and length of the pendant drop (see Fig. 4). The presence of the polymer macromolecules generates a 


\begin{tabular}{lccccccccc}
\hline$c(\mathrm{ppm})$ & 2 & 10 & 20 & 50 & 100 & 250 & 500 & 750 & 1000 \\
\hline$\lambda(\mathrm{ms})$ & 0.096 & 0.76 & 1.13 & 4.5 & 8.6 & 19.6 & 60 & 76.5 & 140 \\
\hline
\end{tabular}

Table 1 Values of relaxation time $\lambda$ for all the polymeric solutions used in this work [38].

\begin{tabular}{lccccc}
\hline Type of feeding element & capillary & capillary & beveled needle & capillary & capillary \\
\hline Element outer diameter $(\mathrm{mm})$ & 0.7 & 1.27 & 1.64 & 2.80 & 4.80 \\
\hline Drop diameter resulting $D(\mathrm{~mm})$ & 2.96 & 3.28 & 3.69 & 4.06 & 5.01 \\
\hline
\end{tabular}

Table 2 Feeding elements used to form the drops.

quasi-cylindrical filament, which takes more time to thin and pinch off $[44,45]$. Using a beveled needle the drop slips down to the needle tip and detaches from the outer hydraulic radius in the tip, so the travel height (and size) of the drop is practically the same as that of its newtonian counterpart.

\section{Results}

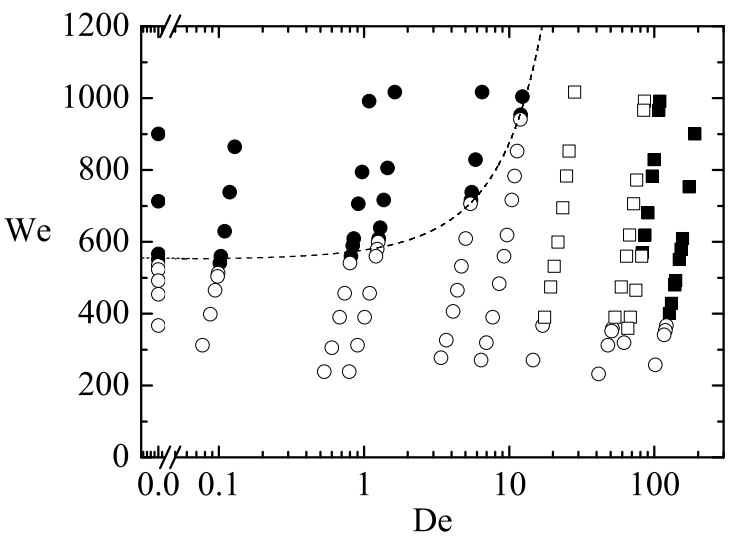

Fig. 5 Drop impact modes as a function of We and De numbers: prompt splash (black circles), no splashing (white circles), no splashing with clear levitated ejecta sheet (white squares), and "fingers splashing" during the spreading (black squares). The dashed line marks the limit or transition from no splash to prompt splash. Re varies in the range of 120015700 for all the realizations. Each quasi-vertical line drawn by the symbols correspond to one different concentration $c$ used in this work, from 0 to $1000 \mathrm{ppm}$ (from the left to the right, respectively).

The results of several experimental runs alowed us to produce a regime diagram in the $W e-D e$ plane. For this purpose, the normal impact velocity $u_{0}$ of a drop with $D=3.28 \mathrm{~mm}$ and the polymeric concentration $c$ of the liquid composing the drop were varied in a relatively wide range. Fig. 5 and 6 show our results. Four drop impact modes can be clearly distinguished: (white circles) no splashing with a lamella in an apparent contact with the solid surface, (black circles) prompt splash ejecting very small and fast secondary droplets, (white squares) no splashing with clearly levitated ejecta sheet or lamella, and (black squares) "fingers splashing" during the fast spreading stage, i.e., in this case the levitated ejecta sheet destabilised and eventually forms fingers around the ridge, with droplets attached to their tips. In this last mode, a competition between capillary and elastic forces trying to retract the fingers takes place, with the kinetic energy (inertia) promoting the thinning and break up of the fingers, eventually leading to pinch off and droplets detachment, i.e., splash. This could be interpreted as a special type of corona (thin-sheet) splash. The number of secondary droplets ejected is higher as $W e$ and $D e$ increase. This behaviour is comparable to that found by Rozhkov et al. [27] for the impact of polymeric drops on small targets. Interestingly, in Fig. 5 it can be observed a clear transition from prompt splash to suppression of splash and then to this corona splash as De increases, i.e., as the concentration of the polymeric additive in water is increased. This secondary transition need further study, as the scope of this work is on the suppression of prompt splash, but it is possible that the dynamics transition from prompt splash to corona splash could shine some light into the underlying mechanisms separating both types of splashing.

The dashed line in Fig. 5 marks the limit or transition from prompt splash to splash suppression. The threshold increases following an exponential law (in fact, it fits quite well to a exponential function), and for $D e$ around 15 the critical Weber number $W e_{c}$ reaches double the value for pure water $(D e=0)$. The abrupt change to the slope of the limit from no splash to prompt splash could be associated to the transition from dilute to semi-dilute solutions, since the physical structures and behaviours of dilute and semi-dilute polymer solutions are very different from each other. A natural question is whether there is an asymptote for a certain number of $D e$ from which prompt splash is totally suppressed. Unfortunately, experimental limitations did not allow 

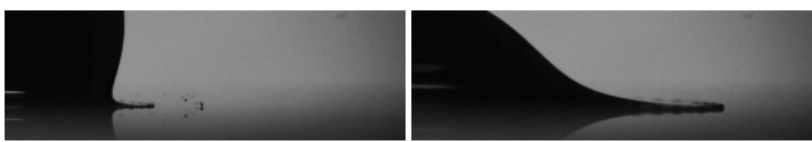

Promp Splash
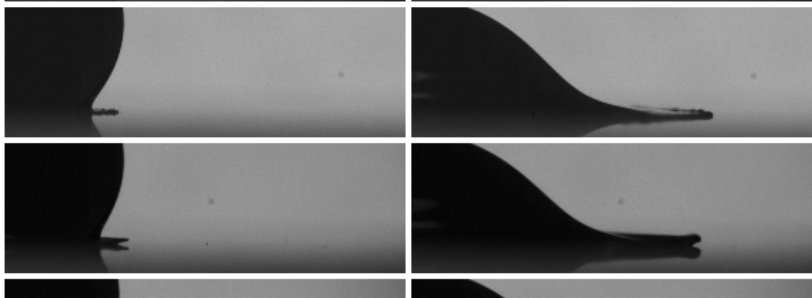

No Splash
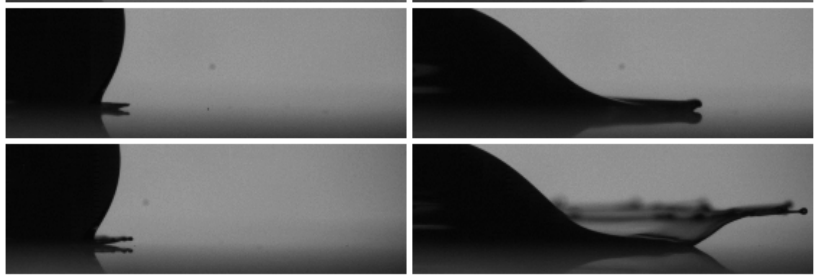

$\square_{\text {No Splash }}^{\text {Smooth lam }}$

$\sim 90 \mu \mathrm{s}$

$\sim 454 \mu \mathrm{s}$

Fig. 6 Illustration of the drop impact modes found in the regions map of the Fig. 5. The parameters characterizing the drop impacting were (from top to bottom): $D e=0.97(c=10 \mathrm{ppm}), D e=10.88$ ( $c=100 \mathrm{ppm}), D e=24.80(c=250 \mathrm{ppm})$, and $D e=96.79$ $(c=750 \mathrm{ppm})$, and $W e=788$ for all the cases.

us to explore $W e$ higher than 1400 . In any case, it is interesting to note that very small amount of this polymeric additive can inhibit the prompt splash. Fig. 5 does not allow to understand the role played by $R e$, independently as $W e$ and $R e$ are somewhat bound together. Therefore, in order to try to reveal the influence of the Reynolds number $R e$, experiments with different drop sizes (ranging the diameters show in Table 2, Sec. 2) were also conducted. Fig. 7 depicts the results of critical Weber number $W e_{c}$ versus $D e$. The dashed line marks the transition shown in Fig. 5. It is observed that from $D e \simeq 5$ (dotted line) all the points show a relatively good agreement with that transition line. It is possible to then conclude that there is a value of $D e$ from which the influence of the $R e$ seems to be negligible, at least within the studied range of $R e$.

In order to understand the the key mechanism behind the suppression of the prompt splash, the evolution of the start of the lamella along the smooth solid substrate was studied in detail. To this end, experiments with larger spatial and temporal resolution were performed, see Sec. 2. We was fixed in order to study the influence of $D e$ alone. Fig. 8 shows the lamella radius $b$ over time $t$ for three cases: $D e=0$ (pure water), $D e=1.41$, and $D e=10.75$, the first two cases with prompt splash and the last one without it. As it can be observed, all the cases are overlapped, i.e., the evolution of the start of the lamella is practically the same. This means that the suppression of prompt splash is not motivated by the loss of energy due to viscous friction, so the viscous contribution of the polymer additive is negligible at this point.

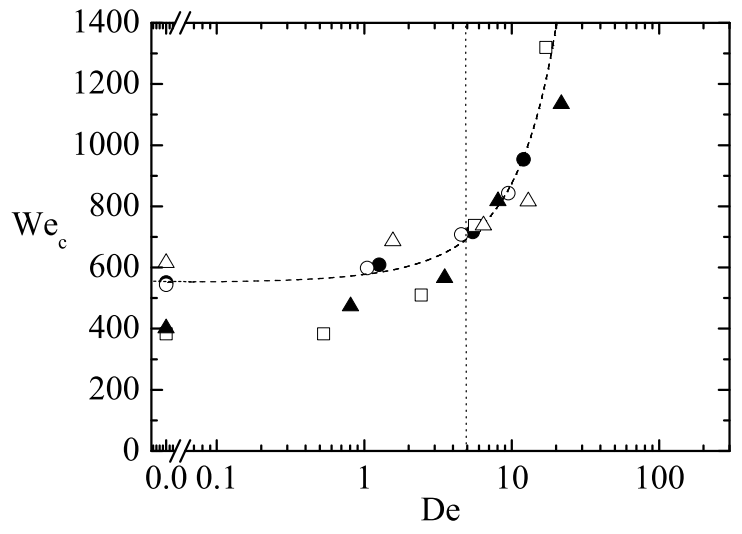

Fig. 7 Critical Weber number $W e_{c}$ versus $D e$ for five different ranges of $R e$ (corresponding to five different drop diameters D): 6580-11420 (white triangles), 7488-11380 (black circles), 7468-11993 (white circles), 6377-10807 (black triangles), and 7641-11738 (white squares). The dashed line marks the limit or transition from no splash to prompt splash for the range of Re 7488-11380 (see Fig. 5). From De $\simeq 5$ (dotted line) all the points show a relatively good agreement with the painted transition line.

Moreover, in order to try to observe the neat effect of the polymeric additives on the splashing dynamics as the concentration is increased, we analysed both the turnover point $a(t, c)$ and the position of the tip of the lamella $b(t, c)$. The results are presented in Fig. 9. From this graph, it becomes clear that the concentration $c$ of PAA has no effect on the turnover point or the dynamics of the bulk of the droplet, as the position of the turnover point for all the studied concentrations agrees very well with Wagner theory prediction (solid curve), $a=\sqrt{3(D / 2) u_{0} t}[46-48]$. However, the effect is clearly 


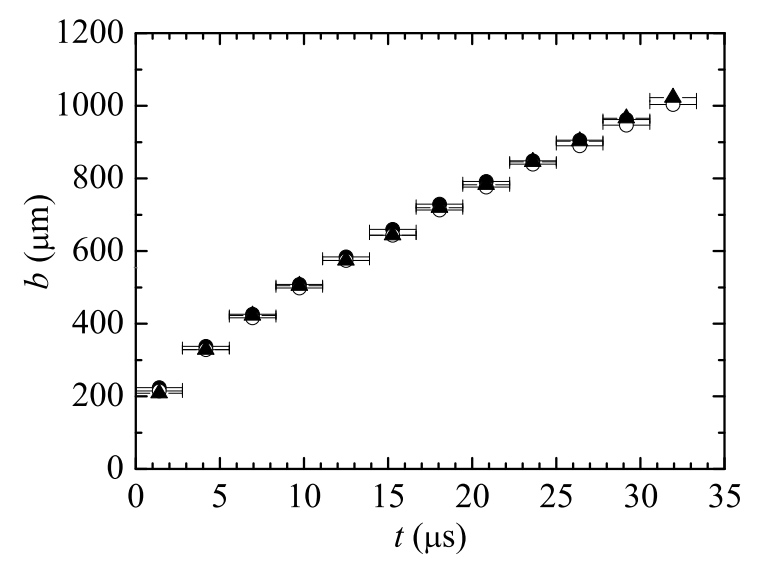

Fig. 8 The start of the lamella travelling along the smooth solid surface, lamella radius $b$ versus time $t$ for: $D e=0$ and $R e=13407$ (pure water, black circles), $D e=1.41$ and $R e=10557$ (white circles), and $D e=10.75$ and $R e=6703$ (black triangles). $W e=764.25$ for all the cases.

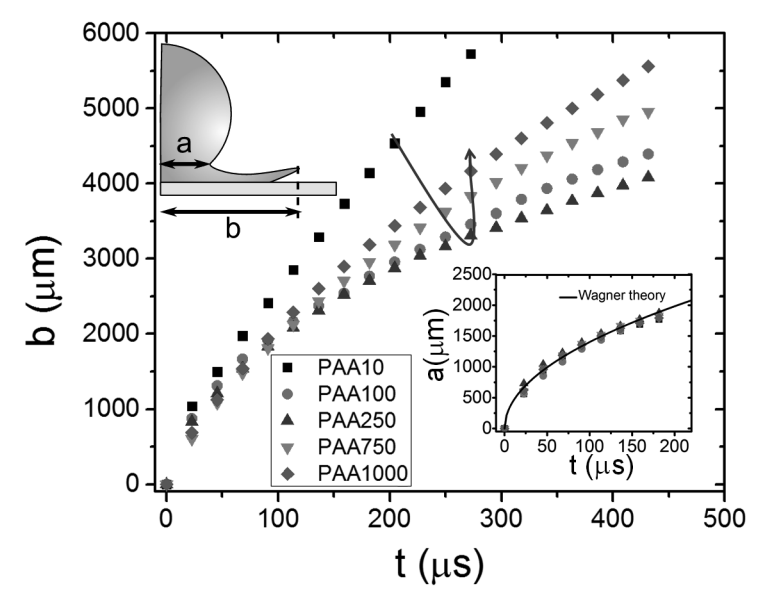

Fig. 9 The radial position of the ejection-sheet tip $b$ (main figure) and the turnover point $a$ (inset) for droplets with constant $W e$ around 715 and varying concentration of PAA $c$. Ejection-sheet velocities is first reduced suppressing splash as $c$ is increased until a limit, after which the ejecta is lifted, leading to an increased tip speed, producing the growth of unstable modes with eventually breakup into a "corona-like" splash. The motion of the turnover point is relatively independent of the concentration of PAA $c$ and agrees well with the Wagner theory prediction (solid curve), $a=\sqrt{3(D / 2) u_{0} t}$.

visible on the position of the ejection-sheet tip. It is possible to observe that the tip of the sheet of the drop with only 10 ppm of PAA travels the fastest, quickly overcoming surface tension and breaking up into droplets. For the case with $100 \mathrm{ppm}$, the ejecta is clearly slowed down, arguably due to the elastic effects of the polymer chains, avoiding splashing. This effect continues and reaches a minimum for $250 \mathrm{ppm}$. As the concentration is increased a secondary effect emerges. The ejected sheet is faster and lifts off, inducing the growth of instabilities which will eventually lead to corona type splash (for the case of $c>750 \mathrm{ppm}$ ), in which the rim at the tip of the sheet destabilized into a finger-like structure and finally breaks up into droplets (see Fig. 6 for better illustration). One could think that the change of behaviour between the cases with 250 ppm and 750 ppm might be associated with the transition from dilute to semi-dilute solutions. However, it is important to point out that both concentrations are far from the overlap concentration $c^{*}=45 \mathrm{ppm}$.

When one analyzes the zoomed-in images of the onset of prompt splash for pure water $(D e=0)$, Fig. 10 (left), it can be seen that small fingers grow from the tip of the lamella ejecting small secondary droplets. However, in Fig. 10 (right) for $D e=10.75$, the retraction of these small fingers is produced. This phenomenon could be explained taking into account the elastic contribution of the polymeric additive. Elastic forces, and not capillary forces, pull the small fingers back, like a spring, preventing the ejection of secondary droplets characteristics of prompt splash and keeping the integrity of the drop impinging on the substrate. The morphology in this dynamical regime is similar to that observed by Bertola [30] in drops impacting onto heated surfaces in the Leidenfrost regime, where a "semi-splash" dynamical regime is reported (with fluid elasticity playing an important role in delaying and suppressing the detachment of satellite droplets), although the phenomenon reported in the current manuscript occurs on a different time scale. Further studies should focus on trying to understand physical similarities within these two situation that could yield a better understanding of the mechanisms underlying this regime.

\section{Conclusions}

In this work, the impact of slightly non-Newtonian drops onto a smooth surface has been studied experimentally. A range of based solutions were prepared by adding small concentrations of high molecular weight polymer such as polyacrylamide (PAA) to a Newtonian solvent (water). A $W e-D e$ map was constructed which clearly shows different types of dynamics, including prompt splash, no splash, and corona-like splash. Particular attention was paid to the effect of polymeric contribution in increasing the threshold (in $W e$ ) for the development of prompt splashing.

It is demonstrated that a very small amounts of such polymer in water (around $0.01 \mathrm{wt} \%$ ) is enough to practically suppress prompt splash. When capillary forces are insufficient to maintain the integrity of the drop, 


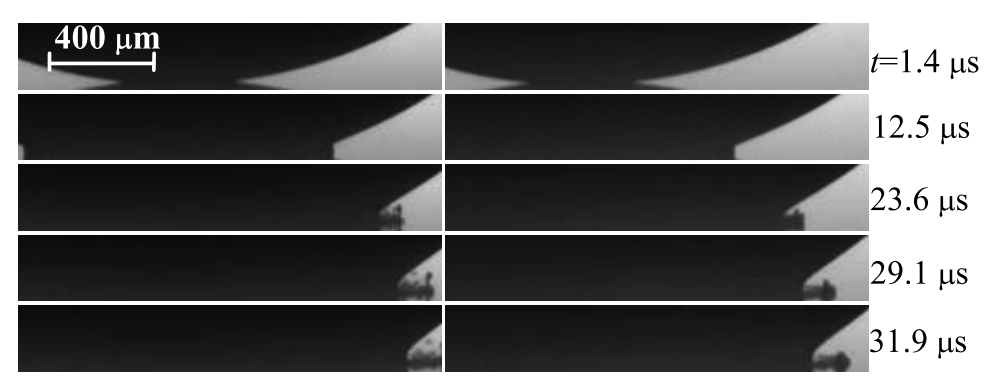

Fig. 10 Zoomed images of the temporal evolution of the start of the lamella travelling along the smooth solid surface for two cases of Fig. 8. The onset of prompt splash of a pure water drop with $D e=0$ and $R e=13407$ (left), and the suppression of the prompt splash for a drop with $D e=10.75$ and $R e=6703$ (right). $W e=764.25$ in both cases.

elastic forces seem to play a role by pulling the wouldbe droplets back to the body of the sheet, preventing their ejection and, therefore, inhibiting prompt splash. An interest effect occurs for higher concentrations of PAA, in which the ejected sheet lifts off, allowing for instabilities to grow and resulting in corona-like disintegration of the drop.

Acknowledgements Partial support from the Spanish Ministry of Science and Education (grant no. DPI2013-46485), Junta de Extremadura (grant no. GR10047), and mobility grant of the program "Jose Castillejo 2015, ref. JC2015-00129" from Spanish Ministry of Education, Culture and Sport is gratefully acknowledged too. AACP received funding from the Royal Society via a University Research Fellowship and from the John Fell Oxford University Press (OUP) Research Fund.

\section{References}

1. B. Derby, Annu. Rev. Mater. Res. 40, 395 (2010)

2. V. Bertola, Recent Patents on Mechanical Engineering 1, 167 (2008)

3. R. Andrade, O. Skurtys, F. Osorio, J. Food Eng. 157, 70 (2015)

4. D. Bolleddula, A. Berchielli, A. Aliseda, Adv. Colloid Interface Sci. 159, 144 (2010)

5. A.L. Yarin, Annu. Rev. Fluid Mech. 38, 159 (2006)

6. C. Josserand, S. Thoroddsen, Annu. Rev. Fluid Mech. 48, 365 (2016)

7. V. Bertola, M. Marengo, Edited by Reinhardt Miller CRC Press pp. 267-298 (2012)

8. S.M. An, S.Y. Lee, Exp. Therm. Fluid Sci. 37, 37 (2012)

9. G. German, V. Bertola, J. Phys.: Condens. Matter 21, $375111(2009)$

10. F. Boyer, E. Sandoval-Nava, J.H. Snoeijer, J.F. Dijksman, D. Lohse, Physical Review Fluids 1, 013901 (2016)

11. S.M. An, S.Y. Lee, Exp. Therm. Fluid Sci. 38, 140 (2012)

12. N. Laan, K.G. de Bruin, D. Bartolo, C. Josserand, D. Bonn, Phys. Rev. Appl. 2, 044018 (2014)

13. M. Guémas, A.G. Marín, D. Lohse, Soft Matter 8, 10725 (2012)

14. V. Bergeron, C. R. Physique 4, 211 (2003)

15. P.A. Williams, R.J. English, R.L. Blanchard, S.A. Rose, L. Lyons, M. Whitehead, Pest Manag. Sci. 64, 497 (2008)
16. V. Bergeron, D. Bonn, J.Y. Martin, L. Vovelle, Nature 405, $772(2000)$

17. R. Crooks, J. Cooper-Whitez, D.V. Boger, Chem. Eng. Sci. 56, 5575 (2001)

18. M.I. Smith, V. Bertola, Phys. Rev. Lett. 104, 154502 (2010)

19. M.I. Smith, V. Bertola, Exp. Fluids 50, 1385 (2011)

20. M.I. Smith, J.S. Sharp, Langmuir 30, 5455 (2014)

21. D. Bartolo, A. Boudaoud, G. Narcy, D. Bonn, Phys. Rev. Lett. 99, 174502 (2007)

22. V. Bertola, Colloid Surf. A-Physicochem. Eng. Asp. 363, 135 (2010)

23. V. Bertola, M. Wang, Colloid Surf. A-Physicochem. Eng. Asp. 481, 600 (2015)

24. H.K. Huh, S. Jung, K.W. Seo, S.J. Lee, Microfluid. Nanofluid. 18, 1221 (2015)

25. D. Izbassarov, M. Muradoglu, Physical Review Fluids 1, $023302(2016)$

26. R. Crooks, D.V. Boger, J. Rheol. 44, 973 (2000)

27. A. Rozhkov, B. Prunet-Foch, M. Vignes-Adler, Phys. Fluids 15, 2006 (2003)

28. V. Bertola, Exp. Fluids 37, 653 (2004)

29. V. Bertola, K. Sefiane, Phys. Fluids 17, 108104 (2005)

30. V. Bertola, Exp. Therm. Fluid Sci. 52, 259 (2014)

31. L. Xu, Phys. Rev. E 75, 056316 (2007)

32. A. Latka, A. Strandburg-Peshkin, M.M. Driscoll, C.S. Stevens, S.R. Nagel, Phys. Rev. Lett. 109, 054501 (2012)

33. J. Palacios, J. Hernández, P. Gómez, C. Zanzi, J. López, Exp. Therm. Fluid Sci. 44, 571 (2013)

34. S.T. Thoroddsen, K. Takehara, T.G. Etoh, J. Fluid Mech. 706, 560 (2012)

35. Y. Liua, P. Tana, L. Xua, PNAS 112, 3280 (2015)

36. C.J. Howland, A. Antkowiak, J.R. Castrejón-Pita, S.D. Howison, J.M. Oliver, R.W. Style, A.A. Castrejón-Pita, Phys. Rev. Lett. 117, 184502 (2016)

37. G. Riboux, J.M. Gordillo, Phys. Rev. Lett. 113, 024507 (2014)

38. P.C. Sousa, E.J. Vega, R.G. Sousa, J.M. Montanero, M.A. Alves, Rheol. Acta 56, 11 (2017)

39. P.C. Sousa, F.T. Pinho, M.S.N. Oliveira, M.A. Alves, Soft Matter 11, 8856 (2015)

40. M.G. Cabezas, A. Bateni, J.M. Montanero, A.W. Neumann, Colloids Surf. A 255, 193 (2005)

41. S.T. Thoroddsen, T.G. Etoh, K. Takehara, N. Ootsuka, Y. Hatsuki, J. Fluid Mech. 545, 203 (2005)

42. N.K. Mishra, Y. Zhang, A. Ratner, Exp. Fluids 51, 483 (2011) 
43. Y. Liu, P. Tan, L. Xu, J. Fluid Mech. 716, R9 (2013)

44. S. Middleman, Chem. Eng. Sci. 20, 1037 (1965)

45. M. Goldin, J. Yerushalmi, R. Pfeffer, R. Shinnar, J. Fluid Mech. 38, 689 (1969)

46. S. Mandre, M.P. Brenner, J. Fluid Mech. 690, 148 (2012)

47. J.M. Kolinski, S.M. Rubinstein, S. Mandre, M.P. Brenner, D.A. Weitz, L. Mahadevan, Phys. Rev. Lett. 108, $074503(2012)$

48. S.D. Howison, J.R. Ockendon, S.K. Wilson, J. Fluid Mech. 222, 215 (1991) 